\title{
Case Report: Radiographic Identification of Intrapleural Misplacement of Epidural Catheter in an Intubated Post-Lung Transplant Patient
}

\author{
Jiang Wu (D) \\ Philip Chung (D) \\ En-Haw $\mathrm{Wu}^{2}$ \\ Kai Zhang' \\ Ryu Komatsu (D) \\ 'Department of Anesthesiology and Pain \\ Medicine, University of Washington \\ Medical Center, Seattle, WA, USA; \\ ${ }^{2}$ Department of Radiology, University of \\ Washington Medical Center, Seattle, \\ WA, USA
}

\begin{abstract}
Intrapleural misplacement of epidural catheter is a rare complication of thoracic epidural placement, which can be difficult to detect in intubated patients with unreliable pain reports and hemodynamic response to the test dose. We describe a case of intrapleural misplacement of thoracic epidural in a 50-year-old man status-post bilateral lung transplant and highlight the use of radiographic techniques to identify the misplacement.
\end{abstract}

Keywords: epidural analgesia, lung transplant, postoperative complications

\section{Introduction}

Thoracic epidural analgesia (TEA) is one of the critical components of Enhanced Recovery after double lung transplant, facilitating earlier extubation and improving post-operative lung expansion after transverse thoracosternotomy, also known as "clamshell" thoracotomy. ${ }^{1-4}$ Some centers advocate for post-operative thoracic epidural placement (TEP) due to the concerns of epidural hematoma during intraoperative full heparinization while on cardiopulmonary bypass. While the risk of epidural hematoma is avoided in this approach, TEP must then be attempted on an intubated and sedated patient post-operatively in the Intensive Care Unit (ICU). In these scenarios, the assessment of TEA becomes challenging due to the lack of patient's pain report and hemodynamic response to test doses may be obscured. We describe a case of intrapleural misplacement of thoracic epidural catheter (TEC) following double lung transplant and present the gross identification of TEC position with bedside chest x-ray (bCXR).

\section{Case Report}

A 50-year-old man with a history of interstitial lung disease underwent bilateral orthotopic lung transplant with transverse thoracosternotomy. His pre-operative CT of the chest demonstrated mild scoliosis, indicating potentially difficult TEP.

He developed significant hemodynamic instability and respiratory insufficiency during the early post-operative period. Subsequently, he was kept intubated and sedated in the ICU sedation protocol with a combination of propofol and fentanyl infusion titrated to the effects. On post-operative day (POD)\#3, TEP to facilitate spontaneous breathing trial prior to extubation was attempted but was aborted because the patient developed severe hypoxemia and hemodynamic instability during patient positioning. On POD\#5, TEP was attempted with the patient in the
Correspondence: Jiang Wu

Email jiangwu@uw.edu 
left lateral decubitus position with a 17-gauge Tuohy needle via the classic landmark-based technique. After two unsuccessful attempts with the right paramedian approach at T5-6 and T6-7 by an experienced attending anesthesiologist, the patient's spine was further flexed and optimized. TEP at T6-7 was reattempted, and loss of resistance (LOR) to saline was achieved at $7.5 \mathrm{~cm}$. A negative aspiration test was obtained with LOR syringe. TEC was threaded through the Tuohy needle and fixed at a depth of $14 \mathrm{~cm}$ relative to the skin. A $3 \mathrm{~mL}$ epidural test dose of $1.5 \%$ lidocaine with 1:200,000 epinephrine was equivocal for positive change in heart rate or blood pressure due to immediate repositioning of patient back to supine. An epidural infusion of $1 / 16 \%$ bupivacaine and $2 \mathrm{mcg} / \mathrm{mL}$ fentanyl was initiated at $6 \mathrm{~mL} / \mathrm{hr}$.

Over the next few days, the patient converted to normal sinus rhythm and was aggressively diuresed but was still unable to be extubated despite incremental increase in epidural infusion rate to $12 \mathrm{~mL} / \mathrm{hr}$ on POD\#8. When sedation was lightened, Critical-care Pain Observation Tools including the items of facial expressions, body movements, compliance with the ventilator, muscle tension, critical-care pain observation score and response to pain interventions were utilized to assess his pain control. The patient was able to follow basic commands and nonverbally endorsed good pain control. However, TEA was difficult to assess due to the confounding of intravenous sedation.

On POD\#9, he developed paroxysmal atrial fibrillation with a rapid ventricular response and new worsening hypoxemia. CT angiogram was obtained on suspicion of pulmonary embolism, which was negative; instead, the CT revealed that the TEC had crossed midline and entered the posterior left pleural space (Figure 1). The catheter was immediately removed, and the decision was made not to reattempt TEP because of continued hemodynamical instability, which lowers the likelihood of significant benefit from TEA now that the patient was 10 days postthoracotomy. Unfortunately, the patient developed hospital-acquired pneumonia (HAP) requiring treatment with broad-spectrum antibiotics, which was further complicated by the development of clostridium difficile colitis. The patient subsequently received tracheostomy for weaning from the ventilator. He was hospitalized for 46 days and required a prolonged recovery at an inpatient rehabilitation facility.

Routine anterior-posterior (AP) bCXR during the patient's ICU stay was retrospectively reviewed
(Figure 2A). Initially, the pathway of TEC was not easily identifiable; however, it could be easily appreciated after digital image enhancement with grayscale inversion (Figure 2B), contrast enhancement by adjusting window and level (Figure 2C), and magnification (Figure 2D) applied to the daily bCXR. After obtaining the optimal contrast and brightness, the images clearly demonstrated the whole path of the epidural catheter in every bCXR for the 4 days between when epidural was placed and CT was obtained. The same methodology was applied to the radiographs taken in a different patient with functional TEP. The resulting radiograph clearly demonstrates the path of TEC (Figure 3).

\section{Discussion}

TEA is considered the "gold standard" analgesia after bilateral orthotopic lung transplant with transverse thoracosternotomy. Compared with systemic analgesics such as opioids, it is superior in providing high-quality and targeted pain relief with minimal medication in addition to its titratability and flexibility in management. ${ }^{1,2}$ Paravertebral catheters (PVCs) have also been utilized but require bilateral and multiple levels of PVC placement to cover a transverse thoracosternotomy incision and chest tube insertion sites to provide comparable analgesia. ${ }^{5}$ Additionally, this approach is more labor intensive, multiple catheters and tubing increase risk of accidental catheter dislodgement, and utilization of multiple infusion catheters carries a higher risk of potential local anesthetic toxicity. ${ }^{6}$ There has not yet been a randomized trial comparing these two modalities. While erector spinae plane block has also been recently promoted as an alternative, its reliability in providing pain coverage in the anterior chest wall is questionable. $^{7}$ The classic landmark-based TEP is still the gold standard technique. Recently ultrasound-guided TEP has been proposed but has not gained widespread acceptance as it is clinically impractical to hold an ultrasound transducer with one hand, while advancing a Tuohy needle with an attached LOR syringe when seeking tactile LOR with the other hand. ${ }^{89}$ Fluoroscopy-guided TEP with epidurography might be the best modality, but its use is limited by positioning difficulty in intubated patients, lack of expertise in anesthesiologists, and inaccessibility to fluoroscopy in most ICU settings.

TEP is classically performed as a landmark-based blind technique with paramedian approach relying on tactile feel using LOR to detect epidural space and unfortunately is associated with a failure rate of $13 \%$ to $32 \%$ in awake 

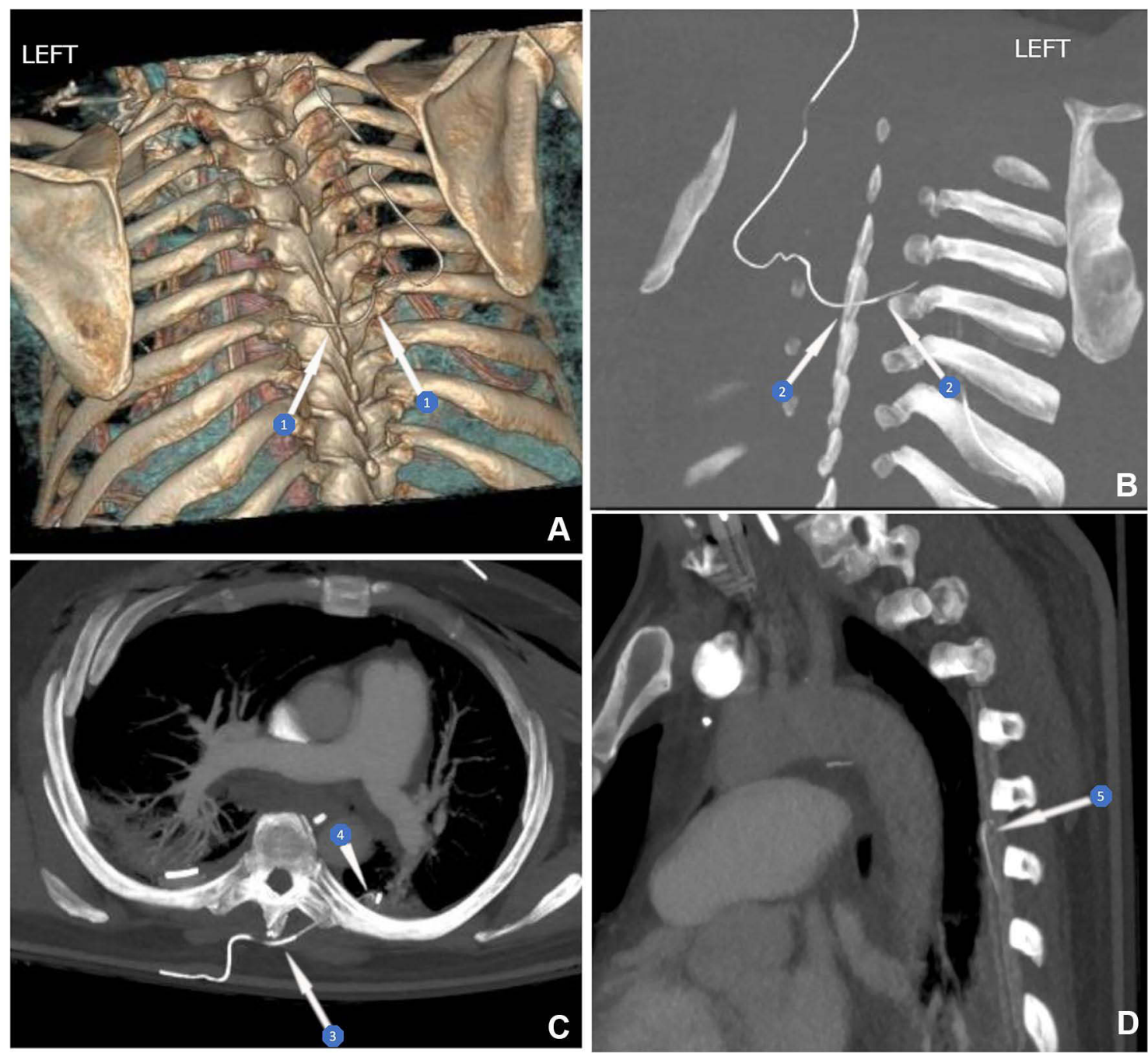

Figure I (A) Volumetric-rendering reconstructed image of the chest CT shows the path of the epidural catheter (arrows labeled \#I) crossing the midline between the T6-7 spinous processes before entering the pleural space via the left 6th-7th intercostal space. (B) Maximal intensity projection (MIP) coronal CT image shows the course of the catheter (arrows labeled \#2) enters the left 6th-7th intercostal space. (C) MIP axial CT image shows the course of the catheter (arrow labeled \#3), crossing the midline and entering the pleural space. Note the tip of the catheter immediately medial the chest tube (arrowhead labeled \#4). (D) MIP sagittal CT image demonstrates the catheter (arrow labeled \#5) anchored at the site of entering the pleural space.

patients in sitting position. ${ }^{10-13}$ Compared to pre-operative TEP in awake and cooperative patients in the sitting position, this case highlights the unique technical challenges in placement and evaluation of post-operative TEP via classic landmark-based technique in an intubated and sedated patient. First, a neutral and flexed thoracic spine is difficult to maintain in lateral decubitus position, predisposing the spine to axial rotation and lateral scoliosis, making blind TEP placement especially challenging. Second, heavy sedation removes the opportunity for patient and/or physician to recognize, report and respond to epidural misplacement and neurological injuries. Intrapleural misplacement typically results in undetectable dermatome coverage and signs of pneumothorax. In our case, the dermatomal assessment was unreliable due to sedation, and the signs of pneumothorax were minimal due to the protection of in situ chest tubes. Third, post-operative hemodynamical instability requiring pressor support, and further hemodynamic perturbances from repositioning between left lateral decubitus and supine obscure and undermine the clinical value of the epidural test dose. In the classical test dose mixture using lidocaine and epinephrine, objective changes in vitals such as hypotension indicate intrathecal or subdural injection, whereas tachycardia suggests intravascular injection. ${ }^{14}$ However, none of these objective measures are reliable because of the aforementioned hemodynamic instability. Subjective patient report of dermatomal coverage or ability to examine motor block from 


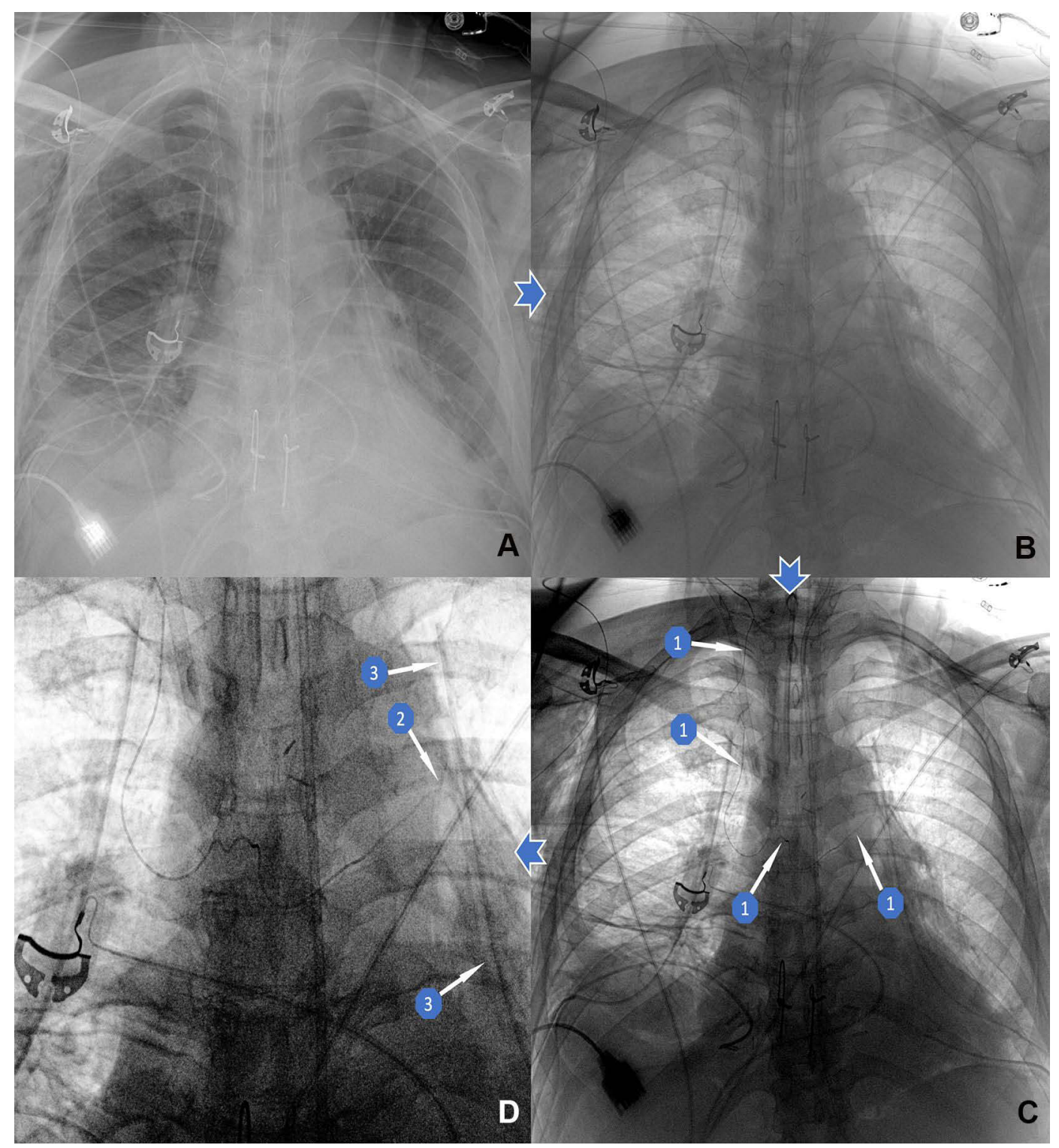

Figure 2 Series of chest x-ray digital image enhancements reveal intrapleural epidural misplacement in our patient. These chest films were obtained on day after epidural placement, 3 days prior to the CT in Figure I. (A) Initial chest radiograph. (B) The same image with grayscale inversion. (C) After adjusting image window and level (digital contrast enhancement), the path of the epidural catheter is better seen (arrows labeled \#I). (D) A magnified crop of the radiograph highlights the path of the epidural catheter crossing midline with tip in the left lung field (arrow labeled \#2) abutting against the left chest tube (arrows labeled \#3).

the test dose is also compromised by heavy sedation. In our case, the clinical response to the epidural test dose was difficult to interpret. Fortunately, we did not have intrathecal, subdural, or intravascular placement of TEC.

To achieve the highest patient safety when providing post-operative TEP, it is vital to identify and confirm the appropriate TEC position following post-operative TEP in intubated post-double lung transplant patients in the ICU. This is especially the case when image guidance is not used for placement, but unfortunately, many traditional strategies for evaluating proper placement are unreliable in this patient population. bCXR is routinely used for evaluation of pulmonary status and can also be useful in confirming epidural position. A retrospective review of this patient's serial CXRs taken in ICU indicated that this intrapleural epidural misplacement could have been detected 3 days earlier (Figure 2). Earlier identification of epidural misplacement may have led to TEC replacement, which may have provided adequate analgesia for effective post-operative pulmonary rehabilitation, extubation, and may have averted development of HAP and the patient's unfortunate prolonged hospital course.

Notably, the digital image enhancement of routine bCXR with grayscale inversion, contrast adjustment, and 


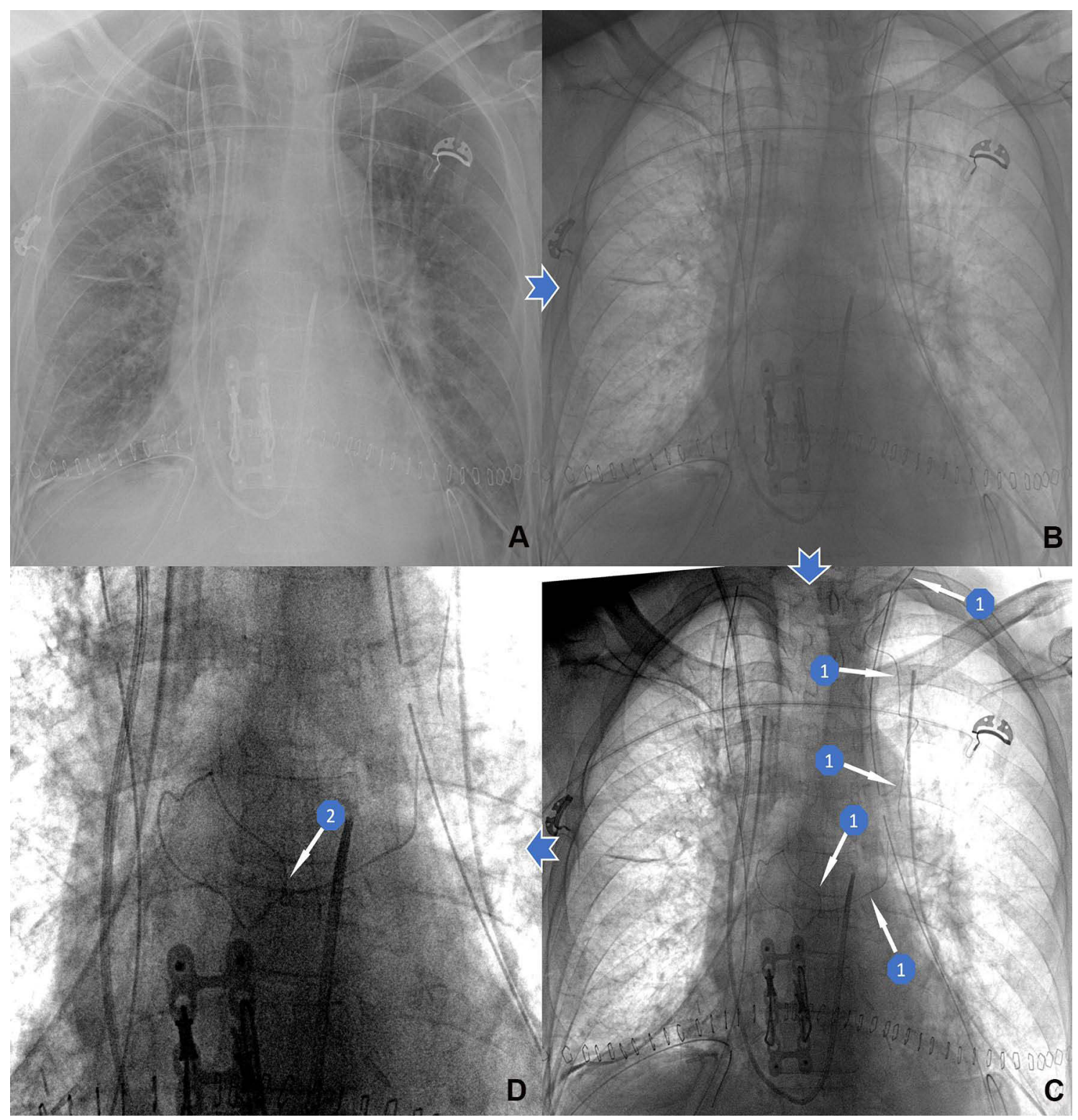

Figure 3 Series of chest $x$-ray digital image enhancements to reveal the appropriate TEP in another bilateral orthotopic lung transplant patient. (A) The initial chest radiograph taken within a day after the epidural placement. (B) The same image with grayscale inversion. (C) After adjusting image window and level (digital contrast enhancement), the path of the epidural catheter is better seen (arrows labeled \#I). (D) A magnified crop of the radiograph highlights the path of the epidural catheter with tip eventually overlying the midline vertebral body (arrow labeled \#2).

magnification significantly improves the visibility of the epidural catheter and tip, which was supported by the examining TEP in a different patient (Figure 3). This finding echoes the conclusions in prior literature, which indicates that radiography can be an effective modality for TEP confirmation. ${ }^{15}$ While prior studies in this area utilized physical films and relied on expert radiologists, the advent of modern digital picture archiving and communication systems (PACS) democratizes bCXR evaluation to both radiologists and bedside practitioners. It is still not a routine practice for radiologists to scrutinize and remark upon TEC position while reviewing daily bCXR. Despite the accessibility of digital images, it is also not common practice for anesthesiologists or acute pain physicians to utilize digital image enhancement of routine bCXR to confirm TEP. This case highlights the clinical value of this tool within the subset of patients receiving a post-operative TEP but remaining sedated in the ICU with hemodynamic instability.

\section{Conclusion}

Our case illustrates the challenges in both post-operative TEP and clinical assessment of TEA in intubated post-lung 
transplant patient. This case highlights the merits of the digital image enhancement of routine bCXR in confirmation of TEP in these difficult placement scenarios when clinical assessment of TEA is unreliable. To support the routine use of this method, a prospective and large trial is needed to detect its diagnostic sensitivity and specificity.

\section{Ethics Statement}

Informed consent, approved by Human Subjects Division of University of Washington, has been provided by the patient to have the case details and any accompanying images published. No additional institutional approval was required to publish the case details. This research activity was conducted in accordance with the Declaration of Istanbul.

\section{Funding}

There is no funding to report.

\section{Disclosure}

The authors report no conflicts of interest in this work.

\section{References}

1. Pottecher J, Falcoz P-E, Massard G, Dupeyron J-P. Does thoracic epidural analgesia improve outcome after lung transplantation? Interact Cardiovasc Thorac Surg. 2011;12(1):51-53. doi:10.1510/ icvts.2010.241489

2. Feltracco P, Barbieri S, Milevoj M, et al. Thoracic epidural analgesia in lung transplantation. Transplant Proc. 2010;42(4):1265-1269. doi:10.1016/j.transproceed.2010.03.109

3. McLean SR, von Homeyer P, Cheng A, et al. Assessing the benefits of preoperative thoracic epidural placement for lung transplantation. $J$ Cardiothorac Vasc Anesth. 2018;32(6):2654-2661. doi:10.1053/j. jvca.2018.04.002
4. Dinic VD, Stojanovic MD, Markovic D, Cvetanovic V, Vukovic AZ, Jankovic RJ. Enhanced recovery in thoracic surgery: a review. Front Med. 2018;5:14. doi:10.3389/fmed.2018.00014

5. Hutchins J, Apostolidou I, Shumway S, et al. Paravertebral catheter use for postoperative pain control in patients after lung transplant surgery: a prospective observational study. $J$ Cardiothorac Vasc Anesth. 2017;31(1):142-146. doi:10.1053/j.jvca.2016.05.006

6. Yeung JHY, Gates S, Naidu BV, Wilson MJA, Gao Smith F. Paravertebral block versus thoracic epidural for patients undergoing thoracotomy. Cochrane Database Syst Rev. 2016;2:CD009121. doi:10.1002/14651858.CD009121.pub2

7. Onishi E, Toda N, Kameyama Y, Yamauchi M. Comparison of clinical efficacy and anatomical investigation between retrolaminar block and erector spinae plane block. Biomed Res Int. 2019;2019:1-8. doi:10.1155/2019/2578396

8. Karmakar MK, Li X, Ho AM-H, Kwok WH, Chui PT. Real-time ultrasound-guided paramedian epidural access: evaluation of a novel in-plane technique. Br J Anaesth. 2009;102(6):845-854. doi:10.1093/ bja/aep079

9. Salman A, Arzola C, Tharmaratnam U, Balki M. Ultrasound imaging of the thoracic spine in paramedian sagittal oblique plane: the correlation between estimated and actual depth to the epidural space. Reg Anesth Pain Med. 2011;36(6):542-547. doi:10.1097/ AAP.0b013e31823217e7

10. Manion SC, Brennan TJ. Thoracic epidural analgesia and acute pain management. Anesthesiology. 2011;115(1):181-188. doi:10.1097/ ALN.0b013e318220847c

11. Gleicher Y, Singer O, Choi S, McHardy P. Thoracic epidural catheter placement in a preoperative block area improves operating room efficiency and decreases epidural failure rate. Reg Anesth Pain Med. 2017;42(5):649-651. doi:10.1097/AAP.0000000000000637

12. McLeod G, Davies H, Munnoch N, Bannister J, MacRae W. Postoperative pain relief using thoracic epidural analgesia: outstanding success and disappointing failures. Anaesthesia. 2001;56 (1):75-81. doi:10.1046/j.1365-2044.2001.01763-7.x

13. Ready LB. Acute pain: lessons learned from 25,000 patients. Reg Anesth Pain Med. 1999;24(6):499-505. doi:10.1016/s1098-7339(99) 90038-x

14. Guay J. The epidural test dose: a review. Anesth Analg. 2006;102 (3):921-929. doi:10.1213/01.ane.0000196687.88590.6b

15. Hendriks GW, Hasenbos MA, Gielen MJ, van Egmond J, Barentsz JO. Evaluation of thoracic epidural catheter position and migration using radio-opaque catheters. Anaesthesia. 1997;52 (5):457-459. doi:10.1111/j.1365-2044.1997.077-az0073.x
International Medical Case Reports Journal

\section{Publish your work in this journal}

The International Medical Case Reports Journal is an international, peer-reviewed open-access journal publishing original case reports from all medical specialties. Previously unpublished medical posters are also accepted relating to any area of clinical or preclinical science. Submissions should not normally exceed 2,000 words or 4

\section{Dovepress}

published pages including figures, diagrams and references. The manuscript management system is completely online and includes a very quick and fair peer-review system, which is all easy to use. Visit http://www.dovepress.com/testimonials.php to read real quotes from published authors. 